\title{
De l'attention à accorder aux aspects narratifs et littéraires en anglais de spécialité : l'exemple du domaine économique
}

of the need to consider narrative and literary aspects in the study of specialised varieties of English: the field of economics as an example

\section{Catherine Resche}

\section{OpenEdition \\ Journals}

Édition électronique

URL : http://journals.openedition.org/asp/4869

DOI : 10.4000/asp.4869

ISSN : 2108-6354

\section{Éditeur}

Groupe d'étude et de recherche en anglais de spécialité

Édition imprimée

Date de publication : 1 novembre 2016

Pagination : $5-25$

ISSN : 1246-8185

Référence électronique

Catherine Resche, « De l'attention à accorder aux aspects narratifs et littéraires en anglais de spécialité : l'exemple du domaine économique », ASp [En ligne], 70 | 2016, mis en ligne le 01 novembre 2017, consulté le 01 novembre 2020. URL : http://journals.openedition.org/asp/4869 ; DOI : https:// doi.org/10.4000/asp.4869

Ce document a été généré automatiquement le 1 novembre 2020.

Tous droits réservés 


\title{
De l'attention à accorder aux aspects narratifs et littéraires en anglais de spécialité : l'exemple du domaine économique
}

\author{
of the need to consider narrative and literary aspects in the study of specialised \\ varieties of English: the field of economics as an example
}

Catherine Resche

\section{Introduction}

1 Le fait de s'intéresser aux liens éventuels entre discours spécialisés et genres littéraires dans le domaine économique peut surprendre, au premier abord. En effet, on pourrait penser que la science économique, soucieuse d'établir sa légitimité en tant que science en choisissant de s'appeler economics dans la deuxième moitié du XIX ${ }^{e}$ siècle, s'est efforcée de gommer tout ce qui pouvait l'avoir apparentée auparavant à une discipline non scientifique, lorsqu'elle relevait encore de la philosophie morale, puis de l'économie politique. Toutefois, si l'on se fie à la définition de la science économique par Alfred Marshall (1920 [1890] : 4) comme « the study of mankind in the ordinary business of life ", on se rend compte que bon nombre d'œuvres de littérature ou de théatre traitent du même thème, même s'il arrive que, dans les œuvres littéraires, les personnages présentés soient parfois des êtres hors du commun. Se pose alors la question de savoir si une science quelconque peut parvenir à un état de pure objectivité et éviter tout élément narratif ou fictionnel. Est-il possible à l'homme de raisonner sans jamais avoir recours à des tropes comme l'analogie ou la métaphore, de partager le fruit de sa réflexion sans recourir à des segments narratifs? S'agit-il de phénomènes marginaux ? Telles sont les questions que nous nous sommes posées. Si la réponse à ces questions est négative, il nous faut alors déterminer quelles sont la nature et la fonction des emprunts à des formes littéraires. 
2 En tant que linguiste, notre sensibilisation à la langue, à la stylistique, à la rhétorique nous conduit tout naturellement à nous intéresser à ces aspects qui, particulièrement en ce qui concerne le domaine économique, traduisent en filigrane l'histoire des idées et nous renseignent sur les positions épistémologiques, les méthodes de raisonnement propres à telle ou telle époque et nous permettent d'élargir et d'enrichir le spectre de nos investigations en matière d'analyse des discours spécialisés et des milieux qui les produisent.

3 Nous évoquons dans un premier temps le cadre théorique qui nous a permis de nous intéresser, dans une perspective panoramique, à un large éventail de manifestations de la narrativité dans le domaine économique. Nous nous intéressons ensuite à la manière dont les moments narratifs sont déclinés, à l'aide d'exemples tirés de notre corpus d'articles de recherche, de manuels et de discours de politique monétaire, en analysant les fonctions que ces segments remplissent au niveau de l'élaboration de la théorie, puis de sa transmission dans divers contextes.

\section{Cadre théorique}

4 Les discours spécialisés, qui sont le produit des acteurs d'un domaine particulier, ne naissent pas ex nihilo. Ils s'inscrivent dans un contexte, dans une histoire, celle de la discipline, mais aussi dans l'histoire d'une époque, d'une société et dans l'histoire des idées. Il est donc vain d'essayer de les analyser en faisant abstraction du passé qui a forgé la culture du domaine. Par conséquent, c'est en tenant compte de l'axe diachronique que cette étude a été menée. Adam Smith étant considéré comme le père de l'économie classique, le XVIII ${ }^{\mathrm{e}}$ siècle nous a semblé constituer un point de repère logique pour mesurer l'évolution de la place et de la forme des moments narratifs dans les discours économiques, même s'il est évident que les préoccupations en matière d'économie remontent à l'Antiquité. Notre objet étant d'inventorier les frontières narratives du domaine, que ce soit au niveau de l'élaboration de la théorie, donc de la démarche scientifique, ou au niveau de la transmission des idées, des pratiques et des décisions, nous avons envisagé un large éventail de manifestations possibles de cette narrativité : récits, histoires, contes, fables, pamphlets, sans oublier le recours aux tropes (allégories, paraboles, ou métaphores).

5 Avant d'exposer le résultat de nos recherches, nous souhaitons préciser un certain nombre d'éléments à propos de ces manifestations discursives. Si nous sommes consciente que les formes narratives que l'on peut trouver dans les textes ou les discours en économie sont à envisager d'une autre manière que si nous analysions un texte littéraire, il n'en reste pas moins utile de nous référer aux auteurs qui se sont intéressés au récit en littérature (Roland Barthes 1966 ; Émile Benveniste 1966 ; Gérard Genette 1969 ; Umberto Eco 1985 ; Paul Ricœur 1986; Jean-Michel Adam 2011; sans oublier Aristote 2002 et Platon 2002). En effet, ces auteurs nous aident à prendre la mesure de la spécificité des éléments narratifs dans les domaines spécialisés qui nous intéressent. Patrick Charaudeau et Dominique Maingueneau (2002: 484) nous rappellent que les séquences narratives classiques sont caractérisées par «une succession temporelle d'actions " et une unité d'action; une situation initiale est troublée par un élément ou un événement perturbateur qui créent une certaine tension (c'est le nœud de l'intrigue); l'histoire se termine quand la solution au problème a été 
trouvée et cela correspond au dénouement, à l'issue duquel la nouvelle situation se stabilise.

Il va de soi que les aspects narratifs des discours spécialisés ne correspondent pas point pour point à la description canonique établie à partir des genres littéraires. Mais l'idée de R. Barthes selon laquelle le récit est l'une des grandes catégories de la connaissance que nous utilisons pour comprendre et ordonner le monde laisse à penser que les aspects narratifs ont leur place non seulement dans la démarche scientifique, mais également dans le partage par les scientifiques de leurs découvertes avec leurs pairs ou de la connaissance qu'ils ont de leur domaine avec un public plus large. T. Todorov (1981) nous rappelle que le narrateur a en tête le public auquel il s'adresse et le but qu'il s'est fixé. En fonction de cela, il adaptera les moyens utilisés pour faire passer son message (analogie, métaphore, anecdotes, illustrations, etc.). Au-delà du contenu premier de ce qui est narré, l'intérêt des moments narratifs est également de présenter et de construire une vision du monde, et ce sont parfois des méga-récits qui sont ainsi élaborés et qui peuvent influencer pendant un temps la recherche et l'état des connaissances, jusqu'à ce qu'une autre vision vienne remettre en cause le grand récit et introduise un changement de paradigme (Kuhn 1970).

Considérons, par exemple, la métaphore; certes, elle a longtemps été bannie des sciences au motif qu'elle devait être réservée à la poésie ou la littérature, mais elle permet aussi une démarche heuristique (Boyd 1993 [1979]) et son pouvoir modélisateur ne saurait être nié. D'autres manifestations des ressorts narratifs ne sauraient être négligées, même si elles peuvent paraître moins évidentes et plus éloignées des exemples que l'on peut trouver dans les œuvres littéraires: nous pensons aux graphiques et aux formules qui, à leur manière, racontent aussi une histoire et font également l'objet d'une mise en mots et en récit.

\section{Ressorts narratifs et littéraires dans l'histoire de la pensée et la construction de la discipline}

\subsection{Narrativité et économie politique}

Bien avant que la communauté des chercheurs en économie ne revendique pour leur domaine l'appellation de discipline scientifique, la réflexion sur les problèmes économiques s'était exprimée sous des formes diverses, mais clairement empruntées aux genres littéraires. Ainsi, dès 1705 , Bernard de Mandeville ${ }^{1}$ publiait une fable maintenant considérée comme la fable fondatrice du libéralisme moderne: c'est la «Fable des abeilles" (The Fable of the Bees), qui contient un poème en vers The Grumbling Hive: or, Knaves turn'd honest. La fable suit le mouvement d'un récit, avec une situation initiale où la ruche est prospère, où le penchant de l'élite pour le luxe constitue un moteur pour l'activité économique et où les vices contribuent à l'harmonie de l'ensemble :

A spacious Hive well stockt with Bees

That liv'd in Luxury and Ease;

[...]

Was counted the great Nursery

of Sciences and Industry.

No Bees had better Government,

More Fickleness, or less Content. 
[...]

These Insects lived like Men, and all

Our Actions they perform'd in small:

[...]

Thus every Part was full of Vice,

Yet the hole Mass a Paradise;

[...]

Such were the Blessings of that State,

Their crimes conspired to make 'em Great;

And Vertue, who from Politicks

Had learn'd a Thousand Cunning tricks,

Was, by their happy Influence,

Made Friends with Vice: And ever since,

The worst of all the Multitude

Did something for the Common Good.

Intervient ensuite une complication, qui risque d'entraîner le déclin de la prospérité : afin de devenir vertueux, les habitants de la ruche cherchent à réintroduire l'honnêteté dans le commerce. Le résultat ne se fait pas attendre: le système s'effondre avec le retour de la vertu et tous en subissent les conséquences. Dans cette fable, dans laquelle les abeilles représentent les hommes, c'est la prospérité de l'Angleterre de l' Enlightenment qui est décrite, et Mandeville dépeint l'honnêteté, la décence, ou encore le sens de la hiérarchie comme de fausses vertus, pour mieux démontrer que l'orgueil, la vanité ou la convoitise conduisent à l'opulence. La morale explicite est que les vices privés tendent vers le bien public. Jugée scandaleuse en son temps, notamment par l'Église, dont les attaques furent relayées par la presse qui rebaptisa Mandeville « Mandevil», cette fable annonçait pourtant l'analyse du marché concurrentiel qu'Adam Smith livra dans son célèbre ouvrage de 1776 : An Inquiry into the Nature and Causes of the Wealth of Nations. Sous la forme d'un micro-récit moins polémique, Adam Smith montre, en effet, que chacun, en poursuivant son propre intérêt, œuvre pour le bien commun, même si tel n'est pas son objet initial :

It is not from the benevolence of the butcher, the brewer, or the baker, that we expect our dinner, but from their regard to their own interest. [...] by directing that industry in such a manner as its produce may be of the greatest value, [every individual] intends only his own gain, and he is in this, as in many other cases, led by an invisible hand to promote an end which was no part of his intention. Nor is it always the worse for the society that it was no part of it. By pursuing his own interest he frequently promotes that of the society more effectually than when he really intends to promote it. I have never known much good done by those who affected to trade for the public good. (IV.ii.9)

La métaphore de la "Main invisible ", en tant qu'illustration d'une autre manifestation des aspects littéraires et narratifs des textes économiques, sert de support à la réflexion du penseur et théoricien du domaine. D'aucuns, comme D. Travers (1996) ou L. Wittgenstein (1961) pourraient avancer qu'elle joue un simple rôle de soutien à l'imagination, que, telle une échelle qui permet d'embrasser un horizon plus large, elle peut ensuite être retirée une fois qu'elle a rempli sa fonction, et il est sans doute vrai que bon nombre de chercheurs qui ont eu recours à la métaphore pour formaliser leur recherche oublient de le préciser et ne livrent que le résultat de leur réflexion. Toutefois, lorsqu'elle comble un vide lexical ou permet de partager une découverte avec d'autres chercheurs, elle s'avère essentielle. En l'occurrence, la métaphore de la Main invisible, qui fait désormais partie de la culture commune des spécialistes du domaine, est un petit récit condensé : c'est l'histoire d'un marché qui s'autorégule, 
comme si une main invisible réglait ses mécanismes. Nous sommes à la fois dans un monde fictionnel, mystérieux et presque magique. Nous revenons plus loin sur d'autres fonctions remplies par la métaphore quand nous abordons les segments narratifs dans le cadre de la transmission du savoir.

Il est un autre emprunt au domaine littéraire qui mérite d'être cité : l'essai satirique ou pamphlet, illustré par la " Pétition des fabricants de chandelles » que Frédéric Bastiat rédigea en 1845, s'adressant ironiquement aux Membres de la Chambre des députés pour dénoncer les effets négatifs du protectionnisme et qui fut immédiatement traduit en anglais ${ }^{2}$, d'où son impact outre Manche :

Pétition des fabricants de chandelles, bougies, lampes, chandeliers, réverbères, mouchettes, éteignoirs, et des producteurs de suif, huile, résine, alcool, et généralement de tout ce qui concerne l'éclairage

À MM. les Membres de la Chambre des Députés

Messieurs,

[...] Nous subissons l'intolérable concurrence d'un rival étranger placé, à ce qu'il parait, dans des conditions tellement supérieures aux nôtres, pour la production de la lumière, qu'il en inonde notre marché national à un prix fabuleusement réduit ; car, aussitôt qu'il se montre, notre vente cesse, tous les consommateurs s'adressent à lui, et une branche d'industrie française, dont les ramifications sont innombrables, est tout à coup frappée de la stagnation la plus complète. Ce rival, qui n'est autre que le soleil, nous fait une guerre si acharnée, que nous soupçonnons qu'il nous est suscité par la perfide Albion (bonne diplomatie par le temps qui court !), d'autant qu'il a pour cette île orgueilleuse des ménagements dont il se dispense envers nous. Nous demandons qu'il vous plaise de faire une loi qui ordonne la fermeture de toutes fenêtres, lucarnes, abat-jour, contre-vents, volets, rideaux, vasistas, œils-debœuf, stores, en un mot, de toutes ouvertures, trous, fentes et fissures par lesquelles la lumière du soleil a coutume de pénétrer dans les maisons, au préjudice des belles industries dont nous nous flattons d'avoir doté le pays, qui ne saurait sans ingratitude nous abandonner aujourd'hui à une lutte si inégale. [...] (Sophismes économiques $1845 ; 1^{\text {re }}$ série chapitre VII)

Si Bastiat est davantage cité par les économistes de langue anglaise que par les français, il faut aussi souligner qu'il a été remis au goût du jour par Margaret Thatcher en personne pour qui il était source d'inspiration.

Le même auteur, journaliste et économiste, a également illustré plusieurs principes économiques sous forme de paraboles, comme la parabole de la vitre cassée (1990 [1850]) qui commence ainsi :

Avez-vous jamais été témoin de la fureur du bon bourgeois Jacques Bonhomme, quand son fils terrible est parvenu à casser un carreau de vitre ? Si vous avez assisté à ce spectacle, à coup sûr vous aurez aussi constaté que tous les assistants, fussentils trente, semblent s'être donné le mot pour offrir au propriétaire infortuné cette consolation uniforme : «À quelque chose malheur est bon. De tels accidents font aller l'industrie. Il faut que tout le monde vive. Que deviendraient les vitriers, si l'on ne cassait jamais de vitres?»

La suite de la parabole est l'occasion d'illustrer la distinction entre effets de long terme et effets immédiats en économie, en présentant trois niveaux d'analyse de l'incident. Si l'on considère d'abord l'aspect négatif de la situation, on arrive à une condamnation du geste et on éprouve de la compassion pour le propriétaire du magasin qui doit remplacer la vitre. Si l'on fait une analyse positive de l'incident, on le voit comme un bienfait économique, puisque cela donnera du travail au vitrier et fera tourner la machine économique. Enfin, il ne faut pas négliger l'effet invisible : en dépensant son argent pour remplacer la vitre cassée, le commerçant se ferme la possibilité d'effectuer 
d'autres dépenses, de créer un emploi supplémentaire, de faire avancer la machine économique en investissant. Par analogie avec l'anecdote narrée, la parabole ouvre la voie à la généralisation et à la réflexion.

On pourrait légitimement penser que les emprunts aux techniques littéraires passés en revue jusqu'ici sont caractéristiques d'une époque où l'économie ne s'était pas encore préoccupée de se faire reconnaître en tant que discipline scientifique et où les protagonistes étaient de formation classique, philosophique et littéraire. Il convient donc de vérifier si un changement est intervenu à partir du moment où l'on a commencé à parler de science économique.

\subsection{Narrativité et science économique}

Ce n'est pas un hasard si la discipline économique a voulu s'affirmer comme science en prenant appui sur le modèle scientifique de la physique au XIX ${ }^{e}$ siècle : bon nombre d'économistes étaient alors ingénieurs de formation. Après avoir emprunté un certain nombre de concepts à la physique et se les être appropriés en les adaptant à l'économie ${ }^{3}$, les économistes ont cherché à être autonomes et à délimiter leur terrain d'investigation. La discipline s'est ensuite mathématisée et s'est appuyée sur des statistiques; c'est alors qu'est née l'économétrie, par exemple, sous l'impulsion de Ragnar Frish en 1926. Formules mathématiques et statistiques ont été amplement utilisées pour asseoir la crédibilité des économistes, qui eurent également recours aux graphiques et aux diagrammes pour compenser leur impossibilité à mener des expériences en laboratoire et concevoir des modèles ${ }^{4}$ servant de support à la théorisation. L'on pourrait alors légitimement penser que la scientificité revendiquée irait de pair avec une prise de distance avec les littéraires et un désir fort d'éviter tout emprunt, de près ou de loin, à des manifestations discursives et narratives ou à des figures de style. Toutefois, il semble irréaliste d'envisager que la réflexion scientifique puisse se passer, par exemple, d'analogies ou de métaphores et le simple fait d'avoir pris comme modèle la science physique en apporte la preuve : la vision mécaniste de l'économie, au même titre que la conception biologique de l'économie qui a également imprégné la pensée économique (Resche 2013) soulignent l'existence de métaphores constitutives de la théorie (Boyd 1993 [1979]). Ces deux grandes veines métaphoriques donnent lieu à la constitution de méga-récits selon lesquels l'économie est une machine aux mécanismes réglables ou un organisme vivant dont la croissance est l'objet d'attentions, et qui est sujet à des crises et à des problèmes de santé pour lesquels une solution doit être trouvée. Ces métaphores laissent d'ailleurs à penser, en fonction de ce qu'avance McCloskey (1992 [1990] : 11) à propos de la physique et de la biologie, que la réflexion des économistes repose à la fois sur des métaphores et des histoires: " Among the sciences (in the recent English sense) metaphors dominate physics and stories dominate biology ».

17 Les métaphores jalonnent d'ailleurs l'histoire de la pensée économique: elles permettent aux économistes, théoriciens et chercheurs de voir autrement, de penser autrement, d'imaginer, d'envisager des angles d'approches inédits, de les justifier et de les expliquer auprès de leurs pairs. Elles se matérialisent parfois par la création de machines hydrauliques, comme ce fut le cas pour les inventions de l'économiste Irving Fisher ${ }^{5}$ ou encore de l'ingénieur-économiste Alban William Phillips ${ }^{6}$. Ces machines, qui permettent de visualiser les mécanismes de formation des prix ou l'impact sur 
l'économie d'un certain nombre de facteurs (impôts, importations, exportations, dépenses), font appel à la technique des scénarios: à chaque manipulation possible d'un des leviers, une nouvelle histoire est racontée. À chacun des scénarios possibles correspond, en effet, un récit différent : nous partons d'une situation initiale, le niveau de liquide dans la machine (de liquidités dans l'économie), apportons un changement et envisageons les conséquences. Par exemple, supposons que nous augmentons les impôts; quelle en serait la conséquence sur la dépense des ménages et donc sur l'économie? La conclusion, en forme de dénouement à la nouvelle situation créée, est le juste équilibre à trouver pour ne pas entrainer un ralentissement de la croissance.

Un autre exemple de la place de l'imagination et de la métaphore dans la pensée économique nous est offert par la surchauffe de l'économie (overheating). Cette métaphore est attribuée à Albert Aftalion (Daniel $2010: 264)$ et résulte de l'histoire suivante :

Dans une pièce où il fait froid, on remplit le poêle de charbon. Comme celui-ci s'enflamme lentement, on a tendance à trop en mettre. Conséquence, quand le feu a vraiment pris, il finit par faire trop chaud. On ouvre alors la fenêtre si bien que l'air extérieur ramène la pièce à la température initiale. Et ainsi de suite ... De même en économie, les entreprises, constatant des réserves de pouvoir d'achat, investissent. Ce faisant, elles augmentent non seulement les capacités de production mais également la demande, ce qui les trompe sur la réalité des débouchés existants dans l'économie et les incite à amplifier la dynamique de l'investissement. Résultat, au bout d'un moment, l'économie est confrontée à une surabondance de machines. S'inspirant de son idée du Poêle, Aftalion parle de "surchauffe », expression qui est restée pour désigner les périodes de croissance économique excessive tirée par des niveaux d'investissement trop élevés.

19 Sans entrer dans le détail, car ce serait trop long dans le cadre de cet article, nous pouvons citer un certain nombre d'analogies et de métaphores qui apportent la preuve que des économistes de renom ont intégré la pensée métaphorique à leur réflexion théorique et y ont aussi eu recours pour partager le fruit de leurs recherches avec leurs pairs : par exemple, le concours de beauté de $\mathrm{Keynes}^{7}$, les ciseaux de Marshall ${ }^{8}$, ou encore le commissaire-priseur de Walras 9 .

Par ailleurs, diagrammes et graphiques peuvent aussi être vus comme des représentations métaphoriques: selon les cas, les axes figurent symboliquement niveaux et dates, quantités et prix, et ces représentations racontent une ou des histoires. Leurs auteurs les expliquent et guident le lecteur en le faisant "voyager ", en quelque sorte, dans le paysage du graphique. Les éléments suivants, empruntés à un article de recherche de Paul Ormerod (2015: 9-10), sont assez fréquents et classiques pour être considérés comme un modèle de trame narrative :

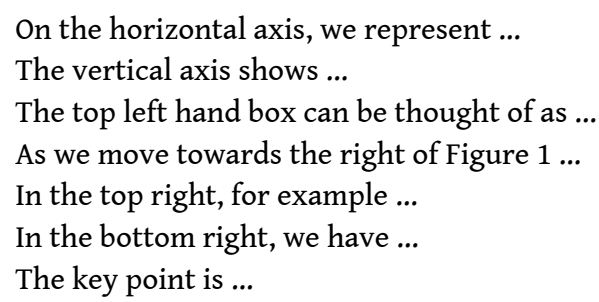

21 Dans les graphiques de type « croix de Marshall », utilisés pour représenter l'offre et la demande, les différents points sont reliés entre eux sous forme de lignes (appelées courbes) et constituent une cartographie du marché, par substitution (McCloskey 2004 [1994] : 63) ; les courbes se croisent en un point d'équilibre, mais il suffit qu'un des 
paramètres change, en abscisse ou ordonnée, pour qu'une nouvelle histoire soit envisagée, une nouvelle situation décrite. Dans un même ordre d'idées, toute formule mathématique raconte une histoire et est souvent explicitée par une petite narration dans laquelle l'auteur précise à quoi correspondent les éléments de la formule et ajoute un commentaire.

Par ailleurs, la rhétorique n'est pas absente des écrits des scientifiques, puisque ces derniers doivent d'abord convaincre leurs pairs du bien-fondé de leur recherche, avant même d'en exposer la méthode et les résultats, ce qui les conduit, par exemple, dans leur introduction, à emprunter à la narration pour brosser un tableau de l'état des lieux de la recherche dans leur domaine, afin de créer leur propre niche de recherche. À ce niveau, ils jouent presque le rôle d'un auteur tiers :

After a period of intensive study of optimal indirect taxation, there has been a renewed interest in recent years in the problem of optimal income taxation, with particular emphasis on capital income taxation and economic growth. (Howitt \& Sinn 1989 : 106, cités par McCloskey 2004 [1994] : 115)

Les segments narratifs ne se limitent toutefois pas aux introductions; ils peuvent également se manifester lorsqu'une expérience est relatée et commentée, comme dans l'extrait suivant (Ormerod 2015: 4-5) : «A computer tournament was organised in which strategies competed in a complex and changing simulation environment. [...] The results of the tournament were a surprise to the organizers ». La conclusion de «l'histoire » est ensuite reformulée ("In other words, [...]») et, en guise de mise en garde, une question est exprimée «An obvious question which follows from this is [...] ».

Il convient également de signaler que, dans l'élaboration de la théorie, un certain nombre d'économistes ont été inspirés par le personnage de Defoe, Robinsoe Crusoe : ce fut le cas, entre autres, de David Ricardo, Francis Isidro Edgeworth, sans oublier que le modèle d'équilibre de long-terme de Ramsey-Cass-Koopmans a pour autre nom le «modèle Robinson Crusoe ».

Qu'elle le reconnaisse ou non, la science économique moderne n'est pas dénuée de segments narratifs et d'emprunts à des formes plus littéraires, que ce soit dans des articles de recherche publiés dans des revues spécialisées et qui, pour convaincre, recourent inévitablement à la rhétorique ou dans des ouvrages, comme le montre George Akerlof (1984) à travers son choix de titre évocateur : An Economic Theorist's Book of Tales. Dans un autre ouvrage co-écrit avec Robert Shiller (2009), G. Akerlof a d'ailleurs reconnu le rôle de la narration et des histoires comme éléments constitutifs de l'économie et de la façon dont elle est perçue :

It is generally considered unprofessional for economists to base their analyses on stories. On the contrary, we are supposed to stick to the quantitative facts and theory - a theory that is based on optimisation, especially optimisation of economic variables [...]. But what if stories themselves move markets? What if these stories of overexplanation have real effects? What if they themselves are a real part of how the economy functions? Then economists have gone overboard. The stories no longer merely explain the facts; they are the facts. (Akerlof \& Shiller 2009 : 54)

Toutefois, les aspects narratifs ne servent pas seulement au niveau de l'élaboration de la théorie et de la recherche; ils sont également mis à profit au niveau de leur transmission, à des fins didactiques et de communication. 


\section{Aspects narratifs et littéraires à visées didactique et communicationnelle}

\subsection{Textes et ouvrages à visée didactique}

Les premiers textes à visée didactique dans le domaine furent le fait de Jane Marcet et de Harriet Martineau dans la première moitié du XIX siècle. Si J. Marcet s'adresse plutôt à un public jeune, $\mathrm{H}$. Martineau écrit pour le public adulte de l'époque, mais les deux auteurs choisissent le genre du conte pour contribuer à instruire leur lectorat en matière d'économie politique. Dans son ouvrage John Hopkins's Notions on Political Economy (1833), J. Marcet met en scène un pauvre métayer qui a du mal à faire vivre sa famille nombreuse et se pose des questions sur la répartition des richesses, le niveau des salaires, le chômage, le libre échange, l'émigration, etc. Ce sont autant de sujets abordés sous forme de petits contes qui l'aident à cheminer dans sa compréhension de ces questions et qui comportent une morale :

In the time of the Fairies, things went on no better than they do at present. John Hopkins, a poor labourer, who had a large family of children to support upon very scanty wages, applied to a Fairy for assistance ...

John Jopkins demande alors à une fée de faire disparaitre toutes les richesses pour tous, sans se rendre compte des conséquences : vie plus dure, ruine, chômage, misère. Au bout d'une semaine, conscient des problèmes qu'il a créés, il retourne voir la fée pour lui demander de rétablir la situation antérieure et tire la leçon de ses erreurs:

[A]s he trudged on, pondering it in his mind, he came to this conclusion:- 'Why then, after all, the rich and the poor have but one and the same interest-that is very strange! I always thought they had been as wide apart as the east is from the west! But now I am convinced that the comforts of the poor are derived from the riches of the rich.' (Essay 1) ${ }^{10}$

De son côté, H. Martineau, dont les vingt-cinq parties des Illustrations of Political Economy furent publiées entre 1832 et 1834, était convaincue qu'en mettant en quelque sorte un enseignement gratuit à la portée des petites gens, elle contribuerait à améliorer leur vie et les aiderait à mieux comprendre les mesures de laissez-faire et à s'y adapter. Les sujets qu'elle aborde sont organisés autour de quatre grands principes (la production, la distribution, l'échange et la consommation) et ses histoires couvrent un large éventail de sujets, que ce soit le libre échange (The Loom and the Lugger), l'endettement (The Farrers of Budge Row), la banque (Berkeley the Banker) ou le syndicalisme et la surpopulation (A Manchester Strike). H. Martineau a d'ailleurs inspiré une autre femmeauteur, Millicent Fawcet (1874), qui a ouvertement reconnu sa dette dans sa préface, mais ses contes (Tales in Political Economy), rédigés pour compléter un manuel d'initiation des jeunes à l'économie politique sorti quatre ans plus tôt, ont eu moins de succès :

It is hoped that these little tales may be of some use to those who are trying to teach Political Economy. I cannot let them go to press without a word of apology to Miss Martineau for my plagiarism of the idea, which she made so popular thirty years ago, of hiding the powder, Political Economy, in the raspberry jam of a story ${ }^{11}$.

Selon Mary Poovey (1995 : 132-133), mêler fiction littéraire et économie à une époque qui les considérait comme deux sphères bien distinctes revenait pour J. Marcet et $\mathrm{H}$. Martineau à faire œuvre de pionnières: "fiction claimed authority over emotional knowledge, while economics claimed authority over empirical knowledge». Si ces contes et 
histoires ont pu tomber dans l'oubli pour la plus grande partie du XXe siècle, ils ont suscité un regain d'intérêt depuis quelques décennies, sans doute en écho à la tendance nouvelle de considérer autrement le langage de l'économie, comme le soulignent $\mathrm{M}$. Osteen et M. Woodmansee (1999: 22) : « a language comprised of tropes, tales and other rhetorical devices that are literary and rhetorical rather than scientific or natural ».

31 On pourrait toutefois penser que ce genre d'ouvrage demeure la marque d'un temps révolu, mais il n'en est rien si l'on considère que certains professeurs d'économie contemporains ont choisi le même genre, celui de la fable ou de la parabole, pour faire passer leur message. Comme le souligne Laurence Seidman, Professeur d'économie à l'Université du Delaware, il est important, à ses yeux, de partager les notions importantes de l'économie avec un public plus large et de l'aider à réfléchir aux questions importantes :

I believe strongly that we academics, immersed in technical work, should occasionally take time out to communicate to a wider audience. After all, if we don't, there are others whose skill at writing far surpasses their knowledge of economics and who will be glad to entertain and mislead that wider audience. So, I've tried to make this serious subject accessible and enjoyable. I hope you find it stimulating. (Seidman 1998 : x)

Dans son ouvrage Economic Parables and Policies, L. Seidman met en scène un certain nombre de personnages (Adam et Ève, le Sénateur Myopie et le vieux Karl, etc.) pour apporter des réponses à des questions portant sur les politiques économiques pour l'avenir, sur la façon d'assurer une meilleure répartition des richesses, sur les impôts, la sécurité sociale, le commerce international, l'environnement, la santé, l'éducation, l'investissement, etc. Les paraboles sont l'occasion de poser les problèmes, d'illustrer différentes possibilités et de cheminer progressivement vers une leçon à tirer. Ainsi, la première parabole (An economist's genesis) suit la trame d'un récit classique (Adam 1992 : 63) : la situation initiale décrit Adam et Ève menant une vie heureuse.

In the beginning, Adam and Eve had no tools. To compensate, God saw to it that the weather and soil never failed them. Initially, Adam and Eve devoted all their working time to growing food. [...] Each year they consumed all the food they produced, and each year, productivity and consumption remained the same. For all we know, Adam and Eve were happy. (Seidman ibid.: 3)

Mais la situation se complique quand Adam, dans un rêve qui le transporte plusieurs siècles plus tard, envisage d'avoir un tracteur qui lui permettrait d'augmenter leur récolte. La situation se complique davantage quand Dieu refuse de leur donner ce tracteur qu'ils devront construire par eux-mêmes. Après avoir réfléchi aux efforts demandés, ils décident d'oublier le tracteur. Mais lors d'une promenade, Adam découvre l'existence d'un autre couple, qui envisage de construire un tracteur en consentant les sacrifices nécessaires pour s'assurer un meilleur avenir et ce nouveau rebondissement force Adam et Ève à réfléchir à nouveau à la meilleure décision à prendre, ce qui donne à l'auteur l'occasion de mener une réflexion mettant en œuvre des concepts économiques.

\subsection{Manuels d'économie et fiction économique didactique}

Les enseignants-chercheurs en économie ne se limitent pas à emprunter aux genres littéraires dans des ouvrages de fiction rédigés à l'attention d'un large public; même 
pour un public plus ciblé d'étudiants en économie, ils font appel à des figures de style et ont recours à des segments narratifs.

Ainsi, les manuels d'économie (Samuelson \& Nordhaus 2005 ; Mankiw 2004 ; Lipsey \& Chrystal 1999) offrent bon nombre d'illustrations et d'exemples fondés sur des microrécits fictionnels, aisément repérables par leurs accroches (Suppose/ Consider/Imagine/ Assume/Say ...) qui engagent résolument le lecteur à concevoir un monde imaginaire, dans lequel il n'y aurait, par exemple, que deux pays, deux ressources à échanger, ou encore deux entreprises :

Imagine that in a particular year, nature smiles on farming. A cold winter kills off the pests; spring comes early for planting; there are no killing frosts; rains nurture the growing shoots; and a sunny October allows a record crop to go to market. At the end of the year, family Jones happily settles down to calculate its income for the year. The Joneses are in for a major surprise: the good weather and bumper crop have lowered their and other farmers' incomes. How can this be? The answer lies in the elasticity of demand for foodstuffs. [...] (Samuelson \& Nordhaus 2005 : 71)

To understand why a monopolist would want to price discriminate, let's consider a simple example. Imagine that you are the president of Readalot Publishing Company. Readalot's best-selling author has just written her latest novel. To keep things simple, let's imagine that you pay the author a flat $£ 2$ million for the exclusive rights to publish the book. Let's also assume that the cost of printing the book is zero. [...] Like any parable, the story of Readalot Publishing is stylized. Yet, also like any parable, it teaches some important and general lessons. In this case, there are three lessons to be learned about price discrimination. (Mankiw 2004: 334-335)

On le voit dans la citation ci-dessus, le recours à la parabole n'est pas absent des manuels d'économie et la parabole de la tragédie des «Commons» est d'ailleurs devenue un classique du genre :

Consider life in a small medieval town. [...] Many of the town's families own flocks of sheep and support themselves by selling the sheep's wool, which is used to make clothing. As our story begins, the sheep spend much of their time grazing on the land surrounding the town, called the Town Common. No family owns the land. Instead, the town residents own the land collectively, and all the residents are allowed to graze their sheep on it. Collective ownership works well because land is plentiful. [...] Everyone in town is happy.

As the years pass, the population of the town grows, and so does the number of sheep grazing on the Town Common. With a growing number of sheep and a fixed amount of land, the land starts to lose its ability to replenish itself. Eventually, the land is grazed so heavily that it becomes barren. With no grass left on the Town Common, raising sheep is impossible, and the town's once prosperous wool industry disappears. Many families lose their source of livelihood. (Mankiw 2004: 231)

Les économistes (Barro 199712 ; Mankiw 2004) ont aussi fait amplement appel au personnage de Robinson Crusoe à des fins pédagogiques, tant il est facile de simplifier les choses en évoquant un seul agent économique pour étudier les choix auxquels il se trouve confronté et illustrer la fonction de production, par exemple :

Robinson Crusoe, as you may recall, is a sailor stranded on a desert island. Because Crusoe lives alone, he catches his own fish, grows his own vegetables, and makes his own clothes. We can think of Crusoe's activities-his production and consumption of fish, vegetables, and clothing-as being a simple economy. By examining Crusoe's economy, we can learn some lessons that also apply to more complex and realistic economies. (Mankiw 2004 : 540-541) 

économiques par le biais des termes métaphoriques, mais également pour transmettre les éléments de culture économique qui servent de socle commun à la communauté. Comme on le sait, la métaphore de la Main invisible est mise à profit à des fins pédagogiques pour transmettre la théorie aux étudiants et permettre de la résumer aisément sans avoir à l'expliciter par une périphrase. Un certain nombre d'autres métaphores associées à des théories dont l'enseignement est incontournable sont ainsi devenues monnaie courante et font partie du socle commun culturel partagé. Le tableau ci-dessous donne une idée de métaphores que tout étudiant doit connaître et qui, comme la Main invisible, condensent une histoire sous-jacente (voir tableau 1).

Tableau 1

\begin{tabular}{|l|l|}
\hline Métaphore & Contexte \\
\hline $\begin{array}{l}\text { Lemons (voitures d'occasion } \\
\text { en mauvais état) }\end{array}$ & Asymétrie de l'information \\
\hline Menu costs & Coût induit par les changements de prix \\
\hline Prisoner's dilemma & $\begin{array}{l}\text { Théorie des jeux (illustre pourquoi la coopération est difficile à } \\
\text { obtenir, même quand elle serait bénéfique) }\end{array}$ \\
\hline $\begin{array}{l}\text { Random Walk (marche } \\
\text { aléatoire des prix) }\end{array}$ & Théorie du marché efficient \\
\hline Shoeleather costs & $\begin{array}{l}\text { En contexte d'inflation, les agents préfèrent ne pas garder trop de } \\
\text { liquidités }\end{array}$ \\
\hline
\end{tabular}

Parallèlement aux manuels classiques, un autre outil pédagogique a été mis à la disposition des étudiants depuis un certain nombre d'années par des professeurs d'économie (William Breit \& Kenneth Elzinga, alias Jevons 1986 [1985], l'homo oeconomicus, 1993, 1998 [1995], 2014; Roberts 2001 [2002]; Wight 2002). Il s'agit de romans dont les sous-titres contiennent d'ailleurs les mentions tale, fable ou romance et dont les auteurs ont décidé de faire passer autrement la théorie économique. Nous avons nommé ce genre «fiction économique didactique » dans une étude antérieure 
(Resche 2004 : 143). La plupart de ces romans suivent une intrigue policière et sont inscrits sur les listes complémentaires de lecture des étudiants de première année ; ils mettent en scène un économiste confronté à un ou des crimes et qui va débusquer leurs auteurs en fondant son raisonnement sur des principes économiques. La lecture de ces ouvrages est agréable et l'intrigue, généralement prenante, met en œuvre les ressorts classiques du récit (situation initiale, problème, complication, rebondissements et dénouement), avec, au passage, les conclusions à tirer quant aux concepts importants grâce auxquels les criminels ont pu être identifiés ou la solution apportée. Ces ouvrages n'ont pas la prétention d'être de la grande littérature, mais leur but est de faire comprendre autrement certains aspects théoriques appliqués à la vie de tous les jours, ou de faire découvrir sous un autre angle les idées de certains penseurs comme Adam Smith ou Ricardo.

\subsection{Discours institutionnel et segments narratifs}

Si l'on considère l'économie réelle, on se rend compte qu'elle dépend en grande partie des "sentiments" des acteurs et que les discours qui s'adressent aux agents économiques ne peuvent ignorer les ressources de la rhétorique, des figures de style, de la narrativité pour faire adhérer le public aux décisions prises ou à prendre, que ce soit au niveau des institutions comme les banques centrales, ou au niveau des entreprises, par exemple. Non seulement les responsables doivent asseoir leur crédibilité en affirmant leur engagement vis-à-vis de ceux, citoyens, employés, clients, qui dépendent de leurs décisions, mais il leur faut convaincre le public que leur politique est fondée et le persuader d'y adhérer. Au niveau de l'entreprise, il s'agit de susciter l'adhésion à des valeurs, de souder les équipes, de les encourager, de reconnaître le bon travail effectué. Les dirigeants, dans les différents contextes, doivent montrer leur capacité d'écoute et d'empathie, d'analyse profonde des situations, et leur aptitude à faire face, à mobiliser, à rebondir. Les segments narratifs sont alors opportunément insérés dans le discours pour lui donner une tonalité particulière.

43 L'extrait ci-dessous, tiré d'un discours de Janet Yellen, illustre bien le recours aux petits récits qui permettent de montrer, derrière la «technicienne» et l'économiste préoccupée par l'impact négatif du chômage sur l'économie, l'être humain sensible aux difficultés de ses concitoyens, et de souligner la «leçon » qu'il faut savoir tirer de ces histoires :

Jermaine Brownlee was an apprentice plumber and skilled construction worker when the recession hit, and he saw his wages drop sharply as he scrambled for odd jobs and temporary work. He is doing better now, but still working for a lower wage than he earned before the recession.

Vicki Lira lost her full-time job of 20 years when the printing plant she worked in shut down in 2006. Then she lost a job processing mortgage applications when the housing market crashed. Vicki faced some very difficult years. At times she was homeless. Today she enjoys her part-time job serving food samples to customers at a grocery store but wishes she could get more hours. [...]

I have described the experiences of [...] Jermaine, and Vicki because they tell us important things that the unemployment rate alone cannot. First, they are a reminder that there are real people behind the statistics, struggling to get by and eager for the opportunity to build better lives. Second, their experiences show some of the uniquely challenging and lasting effects of the Great Recession. Recognizing and trying to understand these effects helps provide a clearer picture of the 
progress we have made in the recovery, as well as a view of just how far we still have to go. pour mieux comprendre l'histoire des idées qui ont forgé une discipline et la façon dont les spécialistes du milieu construisent un cadre à leur réflexion et communiquent sur leurs idées. Il en va d'ailleurs de même pour les discours spécialisés des professionnels. Si l'économie est une science «douce", il ne faut pas croire pour autant qu'une 
sensibilisation aux aspects narratifs et stylistiques ne peut s'appliquer aux sciences « dures ». Un certain nombre de chercheurs (Fries 2016 ; Le Cor 2016; Rowley-Jolivet \& Carter-Thomas 2016; Petit 1997) ont montré que les mathématiques, la physique ou la chimie ont également recours à des figures de style, au récit ou à des segments narratifs.

En offrant ce tour d'horizon, nécessairement incomplet, nous espérons avoir convaincu la communauté des chercheurs en langue de spécialité de l'utilité de poursuivre ce genre d'investigations et de l'approfondir. Il s'agit là d'une autre voie d'accès aux discours des milieux spécialisés, qui permet, pour ce qui est du domaine économique, d'aller, en quelque sorte, des « comptes aux contes » ou de parcourir le chemin inverse "from tables to fables".

\section{BIBLIOGRAPHIE}

ADAM, Jean-Michel. 1992. Les Textes : types et prototypes. Paris : Nathan.

ADAM, Jean-Michel. 2011. Genres de récits. Narrativité et généricité des textes. Paris : L'Harmattan.

AKERLOF, George A. 1984. An Economic Theorist's Book of Tales. Cambridge : Cambridge University Press.

AKERLOF, George A. \& Robert J. SHILlER. 2009. Animal Spirits: How Human Psychology Drives the Economy, and Why It Matters for Global Capitalism. Princeton, NJ : Princeton University Press.

ARISTOTE. 2002. La Poétique. Paris : Les Belles Lettres.

BARRO, Robert, J. 1997. Macroeconomics. $5^{\mathrm{e}}$ édition. Cambridge, MA : MIT Press.

BARTHES, Roland. 1966. « Introduction à l'analyse structurale des récits ». Communications 8.

BASTIAT, Frédéric. 1845. Sophismes économiques. Consulté le 18 décembre 2015. <http://bastiat.org/ $\mathrm{fr} /$ sophismes.html>.

BASTIAT, Frédéric. 1990 [1850]. Ce qu'on voit et ce qu'on ne voit pas. Consulté le 21 décembre 2015. <http://bastiat.org/fr/cqovecqonvp.html\#vitre_cassee>.

BENVENISTE, Émile. 1966. «Structure des relations de personne dans le verbe ». Problèmes de linguistique générale 1, Paris : Gallimard, 225-250.

BOYD, Richard. 1993 [1979]. « Metaphor and theory change: What is 'metaphor' a metaphor for? ». In ORTONY, A. (dir), Metaphor and Thought. Cambridge : Cambridge University Press, 481-532.

ChARAUdeau, Patrick \& Dominique MAInGUENEAU (dir.). 2002. Dictionnaire d'analyse du discours. Paris : Seuil.

DANIEL, Jean-Marc. 2010. Histoire vivante de la pensée économique. Paris : Pearson Education France. DUMEZ, Hervé \& Alain JEUNEMAÎTRE, 2005. «La démarche narrative en économie ». Revue économique 56/4. Presses de Sciences Po (PFNSP), 983-1005.

ÉCHAUDEMAISON, Claude-Danièle. 1993. Dictionnaire d'économie et des sciences sociales. Paris : Nathan. 
Eco, Umberto. 1985 [1972]. Lector in Fabula. Paris : Grasset.

FAWCET, Millicent. 1874. Tales in Political Economy. Londres : Macmillan. Consulté le 10 août 2015. <https://archive.org/stream/talesinpolitica00fawcgoog/talesinpolitica00fawcgoog_djvu.txt>.

FRIES, Marie-Hélène. 2016. « Métaphores et mise en récit : le cas de la biologie synthétique aux États-Unis ». In RESCHE, C. (dir.), La mise en récit dans les discours spécialisés. Collection « Aspects linguistiques et culturels des discours spécialisés ». Berne : Peter Lang, 73-95.

GENETTE, Gérard. 1969. Figures II. Paris : Seuil, Points Essais.

GrEenSPAN, Alan. 2001. The September 11 tragedy and the response of the financial industry. Consulté le 2 novembre 2015. <http://www.federalreserve.gov/boarddocs/speeches / 2001/20011023/>.

HowitT Peter \& Hans-Werner SinN. 1989. « Gradual reform and capital income taxation », American Economic Review 79, 106-124.

JeVons, Marshall. 1986 [1985]. The Fatal Equilibrium. Cambridge, MA \& Londres : MIT Press.

JEVons, Marshall. 1993. Murder at the Margin. Princeton, NJ : Princeton University Press.

Jevons, Marshall. 1998 [1995]. A Deadly Indifference. Princeton, NJ : Princeton University Press.

Jevons, Marshall. 2014. The Mystery of the Invisible Hand: A Henry Spearman mystery. Princeton, NJ : Princeton University Press.

KuHN, Thomas. 1970 [1962]. The Structure of Scientific Revolutions. Chicago : University of Chicago Press.

LE COR, Gwen. 2016. «La mise en récit dans les sciences dures : de l'élaboration théorique à la démonstration mathématique ». In RESCHE, C. (dir.), La mise en récit dans les discours spécialisés. Collection « Aspects linguistiques et culturels des discours spécialisés ». Berne : Peter Lang, 121137.

LIPSEY, Richard, G. \& K. Alec CHRYSTAL. 1999. Principles of Economics. Oxford : Oxford University Press.

MANDEVille (de), Bernard. 1714. The Fable of the Bees. Consulté le 15 novembre 2015. <https:// archive.org/stream/fableofthebeesor027890mbp/fableofthebeesor027890mbp_djvu.txt>.

MANKIW, N. Gregory. 2004. Principles of Economics. Mason, Ohio : Thomson/South Western.

MARCET, Jane. 1833. John Hopkins's Notions on Political Economy. Consulté le 11 novembre 2015 <http://www.econlib.org/library/Marcet/mrcJHo.html>.

MARSHALL, Alfred. 1920 [1890]. Principles of Economics. 8 e édition. Consulté le 19 décembre 2015 <http://www.econlib.org/library/Marshall/marP.html>.

MARTINEAU, Harriet. 2004 [1834]. Illustrations of Political Economy. Peterborough, Ontario, Canada : Broadview Editions.

McCloskey, Donald, N. 1992 [1990]. If You're So Smart. The narrative of economic expertise. Chicago : The University of Chicago Press.

McCloskey, Deirdre, N. 2004 [1994]. Knowledge and Persuasion in Economics. Cambridge: Cambridge University Press. 
ORMEROD, Paul. 2015. « The economics of radical uncertainty », Economics: The Open-Access, openAssessment E-Journal, 9: 1-20. Consulté le 3 janvier 2016 <http://dx.doi.org/10.5018/economicsejournal.ja.2015-41>.

OSTEEN, Mark \& Martha WOODMANSEE (dir.). 1999. « Taking account of the new economic criticism: An historical introduction ». The New Economic Criticism: Studies at the Intersection of Literature and Economics. New York : Routledge, 3-44.

PETIT, Michel. 1997. «Stylistique(s) contrastive(s) du discours scientifique ». ASp 15-18, 139-156.

PlATON. 2002. La République. Paris : Flammarion.

Poovey, Mary. 1995. Making a Social Body: British Cultural Formation 1830-1864. Chicago : University of Chicago Press.

RESCHE, Catherine. 2004. «La théorie économique au détour de la fiction : le roman didactique ». In PETIT, M. (dir.), Aspects de la fiction à substrat professionnel. Bordeaux : Université Victor Segalen Bordeaux 2, Collection Travaux 20.25, 134-151.

RESCHE, Catherine. 2005. «Réflexions à partir d'une métaphore banalisée en économie : la "Main Invisible" d'Adam Smith. Leçons et perspectives ». In Fries, M.-H. (dir.), Métaphores et anglais de spécialité. Bordeaux : Université Victor Segalen Bordeaux 2, Collection Travaux 20.25, 57-76.

RESCHE, Catherine. 2013. Economic Terms and Beyond: Capitalising on the Wealth of Notions. How Researchers in Specialised Varieties of English can Benefit from Focusing on Terms, collection Linguistic Insights 176. Berne : Peter Lang.

RICœUR, Paul. 1986. Du texte à l'action, essais d'herméneutique. Paris : Seuil.

ROBERTS, Russell. 2001. The Choice. A Fable of Free Trade and Protectionism. Upper Saddle River, NJ : Prentice Hall.

ROBERTS, Russell. 2002 [2001]. The Invisible Heart. An Economic Romance. Cambridge, MA \& Londres : MIT Press.

ROWLEY-JOLIVET, Elizabeth \& Shirley CARTER-THOMAS. 2016. « Raconter comment cela se passe vraiment ? Comparaison entre la narration de la recherche dans les cahiers de laboratoire et dans les articles de recherche ». In RESCHE, C. (dir.), La mise en récit dans les discours spécialisés. Collection « Aspects linguistiques et culturels des discours spécialisés ». Berne, Peter Lang, 97-120.

SAMUelson, Paul A. \& William D. NoRdHAUS. 2005. Economics. New York : McGraw-Hill/Irwin SEIDMAN, Laurence S. 1998. Economic Parables and Policies. $2^{\mathrm{e}}$ édition. Armond, NY : M.E Sharpe. SMItH, Adam. 1976 [1776]. An Inquiry into the Nature and Causes of the Wealth of Nations. Campbell, R. H. \& A. S. Skinner (dir). Oxford : Oxford University Press.

TRAVERS, Michael D. 1996. «Programming with agents: New metaphors for thinking about computation ». Massachussetts Institute of Technology. Consulté le 20 décembre 2015 <http:// xenia.media.mit.edu/ mt/ thesis/mt-thesis-2.1.html>.

TODorov, Tzetvan. 1981. Mikhail Bakhtine, le principe dialogique. Paris : Seuil.

WALton, sam. 1993 [1992]. Sam Walton. Made in America. New York : Bantam Books.

WIGHT, Jonathan B. 2002. Saving Adam Smith. A Tale of Wealth, Transformation and Virtue. Upper Saddle River, NJ : Prentice Hall. 
WitTGENSTeIn, Ludwig. 1961. Tractatus Logico-Philosophicus. Trad. D. F. Pears \& B. F. McGuiness.

Londres : Routledge \& Kegan Paul.

\section{NOTES}

1. Né en Hollande en 1670, Bernard de Mandeville avait fait des études de philosophie et de médecine à Rotterdam avant d'émigrer en Angleterre, où il exerça la médecine. Passionné par les fables, il s'est naturellement tourné vers ce genre pour exprimer ses idées.

2. Economic Sophisms, consulté le 12 janvier 2016 <http://www.econlib.org/library/Bastiat/ basSoph3.html>.

3. Voir les concordances établies par Vilfredo Pareto et Irving Fisher (Resche 2013 : 64-65).

4. Nous faisons référence ici à la définition d'un "modèle économique " dans le dictionnaire spécialisé de C.-D. Échaudemaison (1993) : « système abstrait dont la fonction est de représenter la réalité de façon très simplifiée, mais formalisée, ou de permettre l'étude d'un phénomène réel ».

5. «Price machine» de Fisher en ligne, consulté le 12 janvier 2016 <http://priceless-thebook.blogspot.fr/2009/06/price-machine-of-irving-fisher.html>.

6. La machine de Phillips, consulté le 9 novembre 2015 <https://www.youtube.com/watch? $\mathrm{v}=\mathrm{rHIh} 4 \mathrm{YDsZ90}$.

7. Cette métaphore décrit le comportement des investisseurs sur le marché boursier. Les participants au concours doivent choisir, parmi un certain nombre de visages, non pas celui qu'ils jugent le plus beau, mais celui qui recevra le plus grand nombre de suffrages. Le vainqueur est donc celui qui aura le mieux anticipé sur l'opinion majoritaire.

8. Selon la loi de l'offre et de la demande, le prix et la quantité échangée d'un bien sont déterminés par deux forces qui agissent symétriquement à la manière de deux lames d'une paire de ciseaux qui coupent une feuille de papier.

9. Pour illustrer la théorie de l'équilibre général des prix, Walras a recours à la métaphore du commissaire-priseur, qui centralise pour chaque prix le total des offres et des demandes pour chaque produit.

10. <http://www.econlib.org/library/Marcet/mrcJH0.html>

11. <https://archive.org/stream/talesinpolitica0ofawcgoog/talesinpolitica0ofawcgoog_djvu.txt>

12. Le chapitre 2 du manuel de Barro (Macroeconomics) a pour titre : «Work Effort, Production, and Consumption - the Economics of Robinson Crusoe ».

13. En référence au conte bien connu, il s'agit d'une économie définie comme suit : « Neither too cold, nor too hot, just right ».

14. Cette expression a été inspirée par une allusion à un commentaire de journaliste dans un discours prononcé le 19 octobre 1955 par William Mc Chestney Martin, président de la Réserve fédérale du 2 avril 1951 au 31 janvier 1970. Elle a été largement reprise depuis.

\section{RÉSUMÉS}

Cette étude a pour objet de montrer en quoi la prise en compte des segments narratifs et littéraires dans le domaine économique peut bénéficier à la recherche sur les discours et les 
milieux spécialisés qui les produisent. Le cadre théorique est d'abord présenté par rapport à la conception classique de la séquence narrative et la fonction du récit. L'analyse s'attache ensuite à évaluer la place, la nature et la fonction des manifestations narratives et littéraires au niveau de l'élaboration de la théorie économique du XVIII ${ }^{\mathrm{e}}$ siècle à nos jours. L'étude s'intéresse enfin aux segments narratifs qui servent à partager les connaissances et les transmettre à des étudiants, à éduquer un public plus large, ou encore à communiquer (discours officiels des responsables de la politique monétaire). La perspective panoramique adoptée prend en compte un large éventail de genres (récits, contes, fables, paraboles, manuels) et de tropes (allégories, métaphores, analogies), sans oublier les graphiques et schémas qui racontent aussi des histoires.

This paper aims to underline how beneficial a focus on the narrative and literary aspects of economics can be for research into specialised discourse and milieus. The theoretical frame based on the traditional view of narrative and the function of stories is first presented; then, the analysis focuses on the place, the nature and the role of narratives and literary segments in the elaboration of theory from the 18th century onwards. Finally, the paper investigates the narrative segments used for sharing knowledge with students or for educating a broader public, as well as for communicating (via official speeches by the authorities on monetary policy, for example). The panoramic view that is adopted here takes into account a broad range of genres (stories, tales, fables, parables, textbooks) and tropes (allegories, metaphors and analogies), and includes graphs and diagrams that also tell their own stories.

\section{INDEX}

Mots-clés : aspect narratif, communication, domaine économique, élaboration de la théorie, métaphore, partage des connaissances

Keywords : communication, knowledge sharing, metaphor, narrativity, political economy and economics, theory building

\section{AUTEUR}

\section{CATHERINE RESCHE}

Catherine Resche, agrégée d'anglais, est professeur des universités (Paris 2 - Panthéon-Assas), directrice de recherche au Centre de linguistique en Sorbonne (CeLiSo, Paris 4 Sorbonne) et directrice de collection (Peter Lang). Ses recherches portent sur l'anglais de l'économie (terminologie, néologie, métaphores, analyse de discours, de genre, mise en récit). Elle a publié un ouvrage en 2013 (Coll. Linguistic Insights, Peter Lang) Economic Terms and Beyond: Capitalising on the Wealth of Notions et dirigé deux ouvrages collectifs : Terminologie et domaines spécialisés : Approches plurielles (Classiques Garnier) en 2015 et La mise en récit dans les discours spécialisés (Peter Lang) en 2016. catherineresche@club-internet.fr 